\title{
Expression of a manganese peroxidase isozyme 2 transgene in the ethanologenic white rot fungus Phlebia sp. strain MG-60
}

\author{
Yumi Yamasaki ${ }^{1,2}$, Megumi Yamaguchi ${ }^{2}$, Kenji Yamagishi ${ }^{3}$, Hirofumi Hirai ${ }^{4}$, Ryuichiro Kondo ${ }^{5}$, Ichiro Kamei ${ }^{2 *}$ \\ and Sadatoshi Meguro ${ }^{2}$
}

\begin{abstract}
Background: The white-rot fungus Phlebia sp. strain MG-60 was proposed as a candidate for integrated fungal fermentation process (IFFP), which unifies aerobic delignification and semi-aerobic consolidated biological processing by a single microorganism based on its ability to efficiently degrade lignin and ferment the sugars from cellulose. To improve IFFP, the development of a molecular breeding method for strain MG-60 is necessary. The purpose of this study is to establish the transformation method for the strain MG-60 and to obtain the over-expressing transformants of lignin-degrading enzyme, manganese peroxidase.
\end{abstract}

Findings: In the present study, the expression vector regulated by Phlebia brevispora glyceraldehyde-3-phosphate dehydrogenase promoter and terminator was constructed. A polyethylene glycol transformation method for the ethanol-fermenting white-rot fungus Phlebia sp. MG-60 was established with high transformation efficiency, and the manganese peroxidase isozyme 2 gene (MGmnp2) transformants were obtained, showing higher MnP activity than control transformants. MGmnp2 transformants showed higher selective lignin degradation on Quercus wood powder.

Conclusions: This first report of MG-60 transformation provides a useful methodology for widely accessible to interested researches. These results indicate the possibility of metabolic engineering of strain MG-60 for improving IFFP.

Keywords: Lignin; Manganese peroxidase; Phlebia sp. MG-60; White rot fungus

\section{Introduction}

The white-rot fungus Phlebia sp. strain MG-60 was proposed as a candidate for integrated fungal fermentation process (IFFP), which unifies aerobic delignification and semi-aerobic consolidated biological processing by a single microorganism based on its ability to efficiently degrade lignin and ferment the sugars from cellulose (Kamei et al. 2012a, b). To improve this environmentally friendly and unique process for biorefinery of lignocellulose, the development of a molecular breeding method for strain MG-60 is necessary. Manganese peroxidase $(\mathrm{MnP})$ is considered a key enzyme in the lignin degrading basidiomycetous fungi, which catalyzes the oxidation of $\mathrm{Mn}^{2+}$ to $\mathrm{Mn}^{3+}$ along with an $\mathrm{H}_{2} \mathrm{O}_{2}$ generating system (Gold and Alic 1993; Martínez

\footnotetext{
*Correspondence: kamei@cc.miyazaki-u.ac.jp

${ }^{2}$ Department of Forest and Environmental Sciences, Faculty of Agriculture, University of Miyazaki, 1-1 Gakuenkibanadai-nishi, Miyazaki 889-2192, Japan Full list of author information is available at the end of the article
}

2002). Three MnP encoding genes (MGmnp1, MGmnp2 and MGmnp3) were cloned from the strain MG-60 (Kamei et al. 2008). It is expected that the overexpression of $\mathrm{MnP}$ genes improves delignification ability of the strain. In this study, an expression vector was constructed, and transformation efficiency was evaluated. Then, MGmnp2 expressing transformants were generated, and the $\mathrm{MnP}$ activity on the transformants was determined. Additionally, the ability of the transformants to degrade lignin was analyzed.

\section{Materials and methods \\ Construction of expression vectors \\ Phlebia sp. strain MG-60 TUFC40001 (Fungus/Mushroom Resource and Research Center, Tottori, Japan) was main- tained on potato dextrose agar (PDA) plates. Additional file 1: Figure $S 1$ shows the construction procedure for the expression plasmids $\mathrm{p} P b G P D$ (glyceraldehyde-3-}


phosphate dehydrogenase)-HPT, pPbGPD-EGFP and $\mathrm{p} P b G P D-M G m n p 2$. Additional file 1: Table S1 lists the primers (Life Technologies, Carlsbad, CA) used. The PbGPD gene was obtained from Phlebia brevispora HHB-7030 genomic DNA (protein ID: 29450) by PCR amplification with the primers $P b G P D-F 1$ and PbGPD-R1, and the amplified fragment was ligated into the T-Vector pMD20 (TAKARA BIO INC, Shiga, Japan) (steps 1 and 2). The added $A s c$ I restriction enzyme sites, included in the PbGPD promoter and PbGPD terminator, were obtained using the primers $P b G P D-A s c-F 1$ and $P b G P D-A s c-R 1$ (step 3). HPT, EGFP and MGmnp 2 genes containing the added $A s c$ I restriction enzyme site were amplified with PCR by using primers $g H P T-A s c-\mathrm{F} 1, g H P T-A s c-\mathrm{R} 1$, gEGFP-Asc-F1，gEGFP-Asc-R1，gMGmnp2-Asc-F1 and gMGmnp2-Asc-R1, which were designed based on the Escherichia coli hygromycin B phosphotransferase gene (accession number: K01193), the Aequorea victoria EGFP gene (accession number: U55761) and the Phlebia sp. MG-60 MGmnp2 gDNA sequence data (accession number: AB971352), respectively. The amplified HPT, EGFP and MGmnp2 genes were ligated into the expression plasmid after digestion by AscI (New England Biolabs Japan Inc., Tokyo, Japan) according to DNA Ligation Kit Mighty Mix (TAKARA BIO INC, Shiga, Japan) instructions, and then transformed into competent $E$. coli JM 109 (TAKARA BIO INC, Shiga, Japan) for amplification.

\section{Transformation}

Protoplast isolation and polyethylene glycol (PEG)-mediated co-transformation assays with MG-60 were performed in a manner similar to the method reported with Phanerochaete sordida YK-624 previously (Yamagishi et al. 2007). Briefly, MG-60 was pre-cultured in $100 \mathrm{~mL}$ CYM (yeast extract $2 \mathrm{~g} / \mathrm{L}$, polypeptone $2 \mathrm{~g} / \mathrm{L}$, glucose $20 \mathrm{~g} / \mathrm{L}, \mathrm{MgSO}_{4^{-}}$ $7 \mathrm{H}_{2} \mathrm{O} 0.5 \mathrm{~g} / \mathrm{L}, \mathrm{KH}_{2} \mathrm{PO}_{4} 0.46 \mathrm{~g} / \mathrm{L}, \mathrm{K}_{2} \mathrm{HPO}_{4} 1 \mathrm{~g} / \mathrm{L}$, vitamin $\mathrm{B}_{1}$ $1 \mathrm{mg} / \mathrm{L}$, thiabendazole $20 \mu \mathrm{g} / \mathrm{L}, \mathrm{pH} 6.0$ ) medium for $3 \mathrm{~d}$ without shaking. After pre-culture, the mycelia were homogenized for $10 \mathrm{sec}$ and sub-cultured for $3 \mathrm{~d}$ in CYM medium. The mycelia were harvested by filtration and treated with $0.5 \mathrm{M} \mathrm{MgSO}_{4}$ buffer $\left(0.5 \mathrm{M} \mathrm{MgSO}_{4}-7 \mathrm{H}_{2} \mathrm{O}\right.$, $20 \mathrm{mM}$ maleic acid) containing 2.5\% (w/v) Cellulase Onozuka (Yakult, Tokyo, Japan) and 2.5\% (w/v) Lysing Enzymes from Trichoderma (Sigma-Aldrich, MO, USA) at $30^{\circ} \mathrm{C}$ on a shaker NTS-4000B (TOKYO RIKAKIKAI CO, LTD, Tokyo, Japan) at $60 \mathrm{rpm}$ for $4 \mathrm{~h}$. The mycelial suspension was overlaid with $1.0 \mathrm{M}$ SorbOsm $(1.0 \mathrm{M}$ sorbitol, $10 \mathrm{mM}$ MES, pH 6.3) and centrifuged at $1,500 \mathrm{~g}$ in a TOMY LC-122 centrifuge (TOMY SEIKO CO., LTD, Tokyo, Japan) for $20 \mathrm{~min}$. Protoplasts that accumulated at the interface between the two liquid phases were collected, equaling approximately $7.5 \times 10^{6}$ protoplasts per $1 \mathrm{~g}$ of wet mycelial weight. Then, $1.5 \times 10^{6}$ protoplasts in $500 \mu \mathrm{L}$ of $1.0 \mathrm{M}$ SorbOsm were treated with $300 \mu \mathrm{L}$ of DNA solution containing $20 \mu \mathrm{g}$ plasmid in $1 \mathrm{M}$ sorbitol and $0.04 \mathrm{M} \mathrm{CaCl}_{2}$. The tube was placed on ice for $30 \mathrm{~min}$. After treatment, $800 \mu \mathrm{L}$ of $50 \%(\mathrm{w} / \mathrm{v})$ PEG4000 and then $75 \mathrm{~mL}$ regeneration medium were added to the tube. The entire contents were poured onto 50 petri dishes $\left(2.0 \times 10^{4}\right.$ cells $\left./ \mathrm{mL}\right)$, and incubated at $30^{\circ} \mathrm{C}$. Transformants with hygromycin resistance were selected by treatment with $15 \mu \mathrm{g} / \mathrm{mL}$ hygromycin B (Life Technologies, Carlsbad, CA). EGFP and MGmnp2 transformants were selected by genomic PCR amplification with the primers $P b G P D$ prom-F1, gEGFP-Asc-R1 and gMGmnp2-Asc-R1.

\section{MnP activity}

The transformants with high $\mathrm{MnP}$ activities were selected by measuring the activity. Wild type MG-60 (Wt), HPTexpressing transformants (HPT) and MGmnp2-expression candidates (M1 to M14) were cultured in $8 \mathrm{~mL}$ Kirk's high-nitrogen medium (Kirk HN) (Tien and Kirk 1988) at $\mathrm{pH} 4.5$ in test tubes on a reciprocal shaker MMS-3010 shaker (TOKYO RIKAKIKAI CO, LTD, Tokyo, Japan) at $150 \mathrm{rpm}$ in the dark at $28^{\circ} \mathrm{C}$ for 3 or $6 \mathrm{~d}$. After incubation, the whole culture was separated into mycelium and

Table 1 Transformation efficiency of Phlebia sp. strain MG-60 with pPbGPD-HPT, pPbGPD-EGFP and pPbGPD-MGmnp2

\begin{tabular}{|c|c|c|c|}
\hline Transformed plasmid & Hygromycin B & Protoplast regeneration efficiency (\%) ${ }^{a}$ & Co-transformation efficiency $(\%)^{b}$ \\
\hline- & - & 86.7 & - \\
\hline- & + & 0.0 & - \\
\hline pPbGPD-HPT & + & - & 0.042 (549 colonies) \\
\hline pPbGPD-HPT, pPbGPD-EGFP & + & - & 0.029 (380 colonies) \\
\hline pPbGPD-HPT, pPbGPD-MGmnp2 & + & - & 0.038 (489 colonies) \\
\hline \multicolumn{2}{|c|}{ Transformants } & \multicolumn{2}{|c|}{ Transformation efficiency (\%) } \\
\hline \multicolumn{2}{|c|}{ EGFP transformants/HPT transformants } & \multicolumn{2}{|c|}{88.9 (32 clones/36 clones) } \\
\hline \multicolumn{2}{|c|}{ MGmnp2 transformants/HPT transformants } & \multicolumn{2}{|c|}{89.1 (181 clones/203 clones) } \\
\hline
\end{tabular}

pPbGPD-HPT indicates Hygromycin-resistance gene expression plasmid, pPbGPD-EGFP indicate enhanced green fluorescent protein gene expression plasmid, and pPbGPD-MGmnp2 indicate manganese peroxidase isozyme 2 gene expression plasmid derived from MG-60.

${ }^{\mathrm{a}}$ The protoplast regeneration efficiency per protoplast: (regenerated clones/seeding protoplasts).

${ }^{\mathrm{b}}$ The transformation efficiency per protoplast: (HPT transformants/regenerated clones). 
extracellular fluid by centrifugation at $12,000 \mathrm{~g}$ in a centrifuge 5430R (Eppendorf AG, Hamburg, Germany) for $10 \mathrm{~min}$. MnP activity was measured as described by Martínez et al. (1996).

\section{Treatment of wood powder}

The transformants selected above were pre-cultured on PDA plates at $28^{\circ} \mathrm{C}$. The solid medium of Quercus serrata
Thunb.wood powder was adjusted to $80 \%$ moisture content using distilled water and sterilized at $121^{\circ} \mathrm{C}$ for $15 \mathrm{~min}$. One mycelial disk (5 $\mathrm{mm}$ in diameter), punched from colony edges on PDA, were inoculated to the surface of the prepared medium, and then incubated at $28^{\circ} \mathrm{C}$ for $20 \mathrm{~d}$. The test was carried out independently using three flasks per each strain. After incubation, the samples were dried in the oven DX-58 (Yamato Scientific Co., Ltd.,

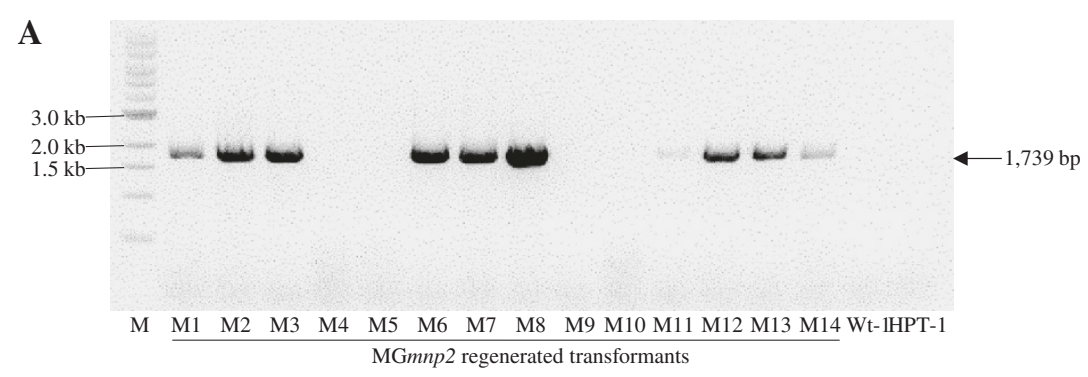

B

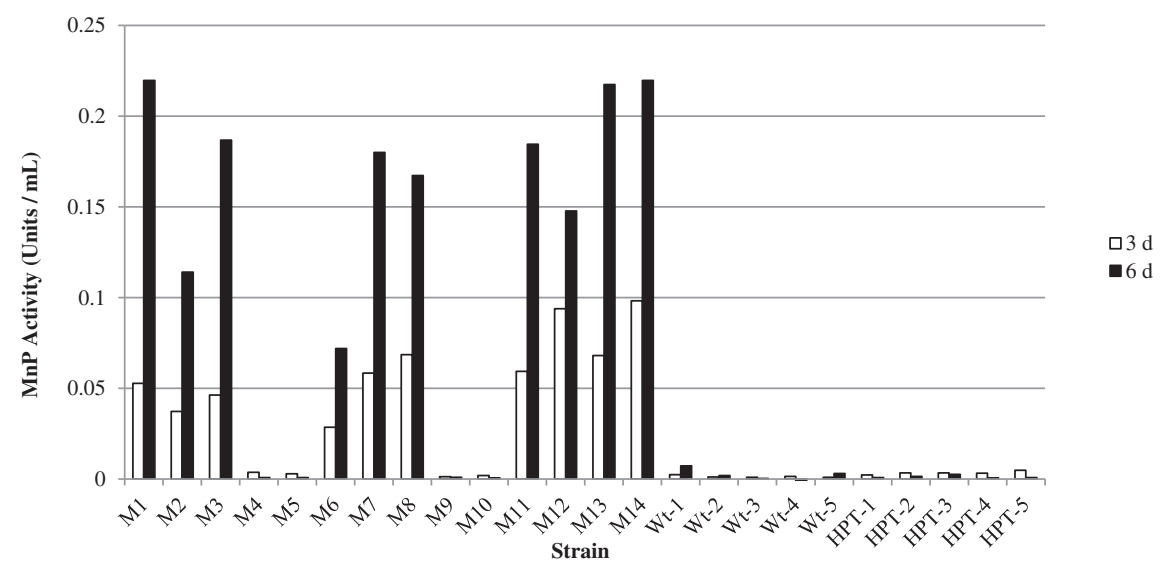

C

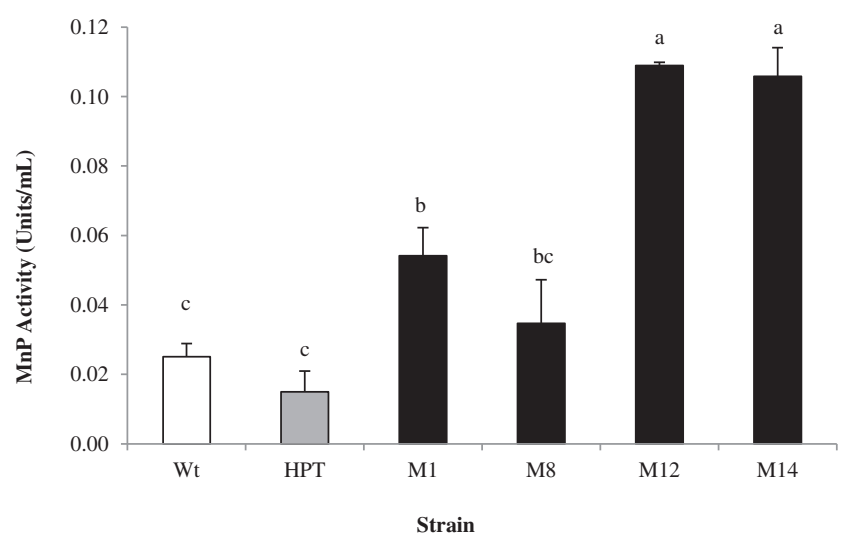

Figure 1 Co-transformation of Phlebia sp. strain MG-60 with pPbGPD-MGmnp2 and pPbGPD-HPT. A, Detection of the MGmnp2 gene in 10 of 14 regenerated protoplasts co-transformed with pPbGPD-HPT and PPbGPD-MGmnp2 by PCR amplification using primers PbGPD-prom-F1 and gMGmnp2-Asc-R1. M indicates a $1 \mathrm{~kb}$ ladder size marker, Wt indicates the wild type, HPT indicates the hygromycin resistant transformant, and M1-M14 indicate MGmnp2 regenerated transformants. B, MnP activity in Kirk's high-nitrogen culture medium under aerobic condition for 3 $\mathrm{d}$ (white column) and $6 \mathrm{~d}$ (black column). One unit of MnP activity was defined as 1 umol reaction product formed per minute. $\mathbf{C}$, MnP activity of various strains in the extracts of Quercus wood powder medium under aerobic condition for $20 \mathrm{~d}$. The tests, using three flasks per strain, were carried out independently. Data are means $\pm \mathrm{SE}(\mathrm{n}=3)$ and values without a common superscript letter are significantly different at $p<0.05$. 
Tokyo, Japan) at $105^{\circ} \mathrm{C}$, and the dry weight was measured. The Klason lignin content of the samples was determined according to the standard analytical laboratory procedure (Sluiter et al. 2011). The selective ability of the transformants to degrade lignin was calculated as the ratio of lignin mass loss to the mass of wood powder (the $L / W$ ratio) (Suhara et al. 2012). To measure the $\mathrm{MnP}$ activity of the transformants, samples were extracted with $20 \mathrm{~mL}$ of $71 \mathrm{mM}$ malonic acid buffer on ice for $1 \mathrm{~h}$ with stirring every $10 \mathrm{~min}$. Data were analyzed by Dunnett's test to evaluate the significance of differences, and $p<0.05$ was regarded as statistically significant.

\section{Results and discussions}

As preliminary experiments, the protoplast regeneration and transformation efficiency of strain MG-60 was measured. The regeneration value on the plate without hygromycin B was $86.7 \%$ (Table 1). The presence of hygromycin B completely inhibited the viability of MG60. The selective marker $\mathrm{p} P b G P D-H P T$ was introduced into the protoplasts of MG-60, and the growth of $H P T$ transformants was observed. On average, approximately 10 colonies per petri dish with hygromycin B showed growth after $7 \mathrm{~d}$ of culturing. The transformation efficiency (Table 1) was higher than that of previous reports with other white rot fungi (Akileswaran et al. 1993; Bartholomew et al. 2001; Yamagishi et al. 2007). These results indicate that the protoplast-PEG method using constructed expression plasmids is viable for the transformation of strain MG-60.

To confirm the antibiotic resistance of the transformants and eliminate the possibility of false-positives, expression of the transgene was confirmed. $\mathrm{p} P b G P D-E G F P$ was used to co-transform strain MG-60 with pPbGPD-HPT, and transformants that contained both plasmids and expressed EGFP were obtained. The expression of $\mathrm{p} P b G P D-E G F P$ could be assessed by the presence or absence of EGFP fluorescence under the microscope. As shown in Table 1, the transformation efficiency of hygromycin-resistant transformants was $0.042 \%$, and EGFP positive transformants accounted for nearly $90 \%$ of the HPT transformants. EGFP fluorescence was observed in the transformants under the microscope (Additional file 1: Figure S2). This is the first study showing that Phlebia sp. strain MG-60 is able to be co-transformed by the protoplast-PEG method.

Phlebia sp. MG-60 was able to degrade lignin and to brighten the unbleached hardwood kraft pulp extensively under hypersaline environment ( $\mathrm{Li}$ et al. 2002). Expression of MGmnp2 was induced under hypersaline condition (Kamei et al. 2008). Therefore, it was expected that overexpression of MGmnp2 improve delignification ability of strain MG-60 under non-saline condition. To obtain MGmnp2 transformants, $14 H P T$ transformants were selected, the insertion of pPbGPD-MGmnp2 was confirmed, and the $\mathrm{MnP}$ activity produced by the each transformant was evaluated. The insertion of pPbGPD-MGmnp2 was observed in 10 of the 14 HPT transformants (Figure 1A). As shown in Figure 1B, the 10 MGmnp2 positive transformants had higher $\mathrm{MnP}$ activities than all $\mathrm{Wt}$ and $\mathrm{p} P b G P D$ MGmnp2 negative transformants in Kirk HN medium. These data indicate that expression of the MGmnp2 transgene was translated successfully into active MnP2.

To evaluate the effects of the expression of the MGmnp2 transgene on wood degradation traits, treatment of Quercus wood powder by selected 4 transformants (M1, M8, M12 and M14) displaying the 2 highest levels of MnP activity at 3 and 6 days culture each was carried out. As shown in Table 2, the mass loss of lignin caused by all four $\mathrm{p} P b G P D$ MGmnp2 transformants was greater than that of $H P T$ transformants. Transformants M12 and M14 showed a significant increase in lignin mass loss compared with the other transformants; however, there was no difference in the weight loss of Quercus wood powder among the transformants. The lignin mass loss to wood powder ratio $(L / W)$ of all four $\mathrm{p} P b G P D-\mathrm{MGmnp} 2$ transformants was higher than that of the HPT transformant, indicating a higher selectivity for lignin degradation. The $L / W$ ratio also revealed a significantly higher value for the M12 and M14 transformants relative to the $H P T$ transformant. Additionally, the MnP activities of the pPbGPD-MGmnp2 transformants on wood powder were higher than those of Wt and the HPT transformant (Figure 1C).

Table 2 Mass loss of Klason lignin and Quercus wood powder, L/W ratio in MG-60 transformants

\begin{tabular}{|c|c|c|c|c|c|}
\hline \multirow[t]{2}{*}{ Strain } & \multicolumn{2}{|c|}{ Transformed plasmid } & \multirow{2}{*}{$\begin{array}{l}\text { Mass loss of Lignin } \\
\text { (\% original mass) }\end{array}$} & \multirow{2}{*}{$\begin{array}{l}\text { Mass loss of wood Quercus } \\
\text { powder (\% original mass) }\end{array}$} & \multirow[t]{2}{*}{$L / W$} \\
\hline & pPbGPD-HPT & $\overline{\mathrm{p} P b G P D-M G m n p 2}$ & & & \\
\hline$\overline{W t}$ & - & - & $31.83 \pm 3.16^{\mathrm{a}}$ & $13.46 \pm 2.41^{\mathrm{a}}$ & $2.43 \pm 0.49^{b}$ \\
\hline HPT & + & - & $11.42 \pm 2.57^{c}$ & $5.59 \pm 0.17^{b}$ & $2.04 \pm 0.68^{b}$ \\
\hline M1 & + & + & $13.93 \pm 2.07^{c}$ & $5.19 \pm 0.17^{b}$ & $2.70 \pm 0.40^{b}$ \\
\hline M8 & + & + & $13.85 \pm 2.40^{c}$ & $5.12 \pm 0.30^{b}$ & $2.69 \pm 0.80^{b}$ \\
\hline $\mathrm{M} 12$ & + & + & $20.88 \pm 2.57^{b}$ & $5.35 \pm 0.12^{b}$ & $3.90 \pm 0.49^{a}$ \\
\hline M14 & + & + & $25.26 \pm 2.02^{\mathrm{b}}$ & $5.95 \pm 0.15^{b}$ & $4.24 \pm 0.26^{a}$ \\
\hline
\end{tabular}

Wt indicates the wild type, HPT indicates the hygromycin resistant transformant, and M1, M8, M12, M14 indicate MGmnp2 regenerated transformants. The tests, using three flasks per strain, were carried out independently. Data means $\pm \mathrm{SE}(\mathrm{n}=3)$ and values without a common superscript letter are significantly different at $p<0.05$. 
However, both the mass losses of wood powder and of lignin were markedly less in the four pPbGPD-MGmnp2 transformants and the HPT transformant than in the Wt (Table 2). This may be due to the delayed growth of transformed strains on wood. While there was no difference in the growth rate between Wt and transformed strains on PDA and Kirk media, the growth rate of transformed strains on Quercus wood powder medium was markedly slower (Additional file 1: Table S2). Even so, the $L / W$ ratio of the M14 transformant was significantly higher than that of Wt. These results suggest that the enhancement of MnP2 activity promotes Klason lignin degradation.

There is a possibility that the decreased ability of transformants to decompose wood was caused by protoplast generation. Many basidiomycete fungi exist as dikaryons in which the two genomes exist in separated nuclei. In previous studies, it has been indicated that the nuclei number was changed during the preparation of protoplasts from dikaryotic mycelia of basidiomycetes (Ohmasa et al. 1987). It has also been reported that the characteristics of progenitor basidiomycetes became altered by protoplast isolation, including growth rate changes (Clark and Anderson 2004). In our study, there was some variation in the sizes of protoplasts prepared from MG-60, and the number of nuclei stained by propidium iodide also varied among the protoplasts (data not shown). It might occur possible that the change in the number of nuclei by protoplast isolation transmuted the original characteristics of MG-60 with regards to delignification; however, it was unclear whether protoplasts of MG-60 were haploid or diploid because the clamp connection was not observed in MG-60.

In summary, our findings demonstrate that the MG-60 strain is able to be co-transformed, and the transformants have MGmnp2 transgene expression. This MGmnp2 expression induced an acceleration of lignin degradation, while the original ability to degrade lignin was decreased by protoplast regeneration of the MG-60 strain. Based on these findings, we continue to select protoplast regeneration strains that had a higher ability to degrade lignin than Wt and to analyze whether there is a correlation between the change in delignification and the number of nuclei.

\section{Additional file}

Additional file 1: Table S1. Oligonucleotides used as primers in this study. Table S2. Mycelial elongation of Phlebia sp. strain MG-60 on PDA or Quercus wood powder media. Figure S1. Construction of HPT, EGFP and MGmnp2 expression plasmids. The procedure used to construct pPbGPD-HPT, pPbGPD-EGFP and pPbGPD-MGmnp2 is described in the text. The horizontal arrows indicate the location and directions of primers. Boxes indicate genes. Figure S2. Confirmation of EGFP fluorescence of EGFP transformants in Phlebia sp. strain MG-60. The EGFP fluorescence of EGFP transformants was observed using a BX51 OLYMPUS fluorescence microscope system.

\section{Abbreviations}

MnP: Manganese peroxidase; HPT: Hygromycin- resistance gene; EGFP: Enhanced green fluorescent protein gene; GPD: Glyceraldehyde-3-phosphate dehydrogenase.

\section{Competing interests}

The authors declare that they have no competing interests.

\section{Authors' contributions}

YY, MY, IK performed the experiments. YY, KY, HH, RK, IK, SM conceived and designed the experiments. YY, IK wrote the paper. All authors read and approve the final manuscript.

\section{Acknowledgments}

This work was supported by Grants-in-Aid for Scientific Research (A) (Nos. 21248023 and 24248030) from the Ministry of Education, Culture, Sports, Science and Technology of Japan.

\section{Author details}

${ }^{1}$ Center of Community Organization, University of Miyazaki, 1-1 Gakuenkibanadai-nishi, Miyazaki 889-2192, Japan. ²Department of Forest and Environmental Sciences, Faculty of Agriculture, University of Miyazaki, 1-1 Gakuenkibanadai-nishi, Miyazaki 889-2192, Japan. ${ }^{3}$ Planning and Promotion Section, NARO Tohoku Agricultural Research Center for Tohoku Region, Morioka 020-0198, Japan. ${ }^{4}$ Department of Applied Biological Chemistry, Faculty of Agriculture, Shizuoka University, Shizuoka 422-8529, Japan. ${ }^{5}$ Department of Agro-Environmental Sciences, Faculty of Agriculture, Kyushu University, Fukuoka 812-8581, Japan.

Received: 22 September 2014 Accepted: 17 November 2014 Published: 27 November 2014

\section{References}

Akileswaran L, Alic M, Clark EK, Hornick JL, Gold MH (1993) Isolation and transformation of uracil auxotrophs of the lignin-degrading basidiomycete Phanerochaete chrysosporium. Curr Genet 23:351-356

Bartholomew K, Dos Santos G, Dumonceaux T, Charles T, Archibald F (2001) Genetic transformation of Trametes versicolor to phleomycin resistance with the dominant selectable marker shble. Appl Microbiol Biotechnol 56:201-204

Clark TA, Anderson JB (2004) Dikaryons of the basidiomycete fungus Schizophyllum commune: evolution in long-term culture. Genetics 167:1663-1675

Gold MH, Alic M (1993) Molecular biology of the lignin-degrading basidiomycete Phanerochaete chrysosporium. Microbiol Rev 57:605-622

Kamei I, Daikoku C, Tsutsumi Y, Kondo R (2008) Saline-dependent regulation of manganese peroxidase genes in the hypersaline-tolerant white rot fungus Phlebia sp. strain MG-60. Appl Environ Microbiol 74:2709-2716

Kamei I, Hirota Y, Mori T, Hirai H, Meguro S, Kondo R (2012a) Direct ethanol production from cellulosic materials by the hypersaline-tolerant white-rot fungus Phlebia sp. MG-60. Bioresour Technol 112:137-142

Kamei I, Hirota Y, Meguro S (2012b) Integrated delignification and simultaneous saccharification and fermentation of hard wood by a white-rot fungus, Phlebia sp. MG-60. Bioresour Technol 126:137-141

Li X, Kondo R, Sakai K (2002) Studies on hypersaline-tolerant white-rot fungi. I: screening of lignin-degrading fungi in hypersaline conditions. J Wood Sci 48:147-152

Martínez AT (2002) Molecular biology and structure-function of lignin-degrading heme peroxidases. Enzyme Mcrob Technol 30:425-444

Martínez MJ, Ruiz-Dueñas FJ, Guillén F, Martínez AT (1996) Purification and catalytic properties of two manganese peroxidase isoenzymes from Pleurotus eryngii. Eur J Biochem 237:424-432

Ohmasa M, Abe Y, Furukawa H, Taniguchi M, Neda H (1987) Preparation and culture of protoplasts of some Japanese cultivated mushrooms. Bull Prod Res Inst 343:155-170

Sluiter A, Hames B, Ruiz R, Scarlata C, Sluiter J, Templeton D, Crocker D (2011) Determination of structural carbohydrates and lignin in biomass. Laboratory analytical procedure NREL/TP-510-42618

Suhara H, Kodama S, Kamei I, Maekawa N, Meguro S (2012) Screening of selective lignin-degrading basidiomycetes and biological pretreatment for enzymatic hydrolysis of bamboo culms. Int Biodeterior Biodegradat 75:176-180 
Tien M, Kirk TK (1988) Ligninperoxidase of Phanerochaete chrysosporium. Methods Enzymol 161:238-249

Yamagishi K, Kimura T, Oita S, Sugiura T, Hirai H (2007) Transformation by complementation of a uracil auxotroph of the hyper lignin-degrading basidiomycete Phanerochaete sordida YK-624. Appl Microbiol Biotechnol 76:1079-1091

doi:10.1186/2193-1801-3-699

Cite this article as: Yamasaki et al:: Expression of a manganese peroxidase isozyme 2 transgene in the ethanologenic white rot fungus Phlebia sp. strain MG-60. SpringerPlus 2014 3:699.

Submit your manuscript to a SpringerOpen ${ }^{\circ}$ journal and benefit from:

- Convenient online submission

- Rigorous peer review

- Immediate publication on acceptance

- Open access: articles freely available online

- High visibility within the field

- Retaining the copyright to your article 